\title{
TOWARD A CLASSIFICATION OF DYNAMICAL SYMMETRIES IN CLASSICAL MECHANICS
}

\author{
GEOfF Prince
}

\begin{abstract}
A one-parameter group on evolution space which permutes the classical trajectories of a Lagrangian system is called a dynamical symmetry. Following a review of the modern approach to the "symmetry-conservation law" duality an attempt is made to classify such invariance groups according to the induced transformation of the Cartan form. This attempt is fairly successful inasmuch as the important cases of Lie, Noether and Cartan symmetries can be distinguished. The theory is illustrated with a presentation of results for the classical Kepler problem.
\end{abstract}

\section{Introduction}

The last few years have seen considerable progress in the understanding of the relation between the symmetries (one parameter-invariance groups) and the conserved quantities of Lagrangian and Hamiltonian systems (see Sarlet and Cantrijn [7] for a review and references). Extensions to Lie's theory of ordinary differential equations and their application to generally non-Lagrangian systems are less well known (see Prince [5]) although the overlap between the two areas is considerable. I am concerned here with invariance groups of Lagrangian systems, not only those arising from Noether's Theorem and its "Cartanian" counterpart, and their relation

Received 4 August 1982. The author is grateful, as always to Willy Sarlet for stimulating discussions especially concerning the details in the Appendix. 
to conserved quantities and the Lagrangian itself. The thrust of the paper is an attempt to classify the invariance groups according to their transformation laws for the Cartan form. The motivation for this is two-fold: firstly, these laws are straightforward for some important classes of symmetries and this inspires a more general exploration. Secondly, the classical results in Riemannian geometry concerning the transformation properties of the metric under various geometrical actions suggest that the corresponding geometric object in Lagrangian mechanics may have interesting transformation properties under dynamical actions.

I have included only the minimum differential geometric background needed for the considerations here and the reader is referred, for example, to the two references already cited along with Estabrook [2] for more details.

\section{Introduction}

I will be considering the symmetries and conservation laws of a Lagrangian system with an $n$-dimensional configuration space $M$. Local co-ordinates $\left(x^{1}, \ldots, x^{n}\right)$ will be used for points in $M$. The system will, in general, be non-autonomous with Newtonian time $t$ and it will be useful to use $M \times \mathbb{R}$ with local co-ordinates $\left(x^{\perp}, \ldots, x^{n}, t\right)$. The evolution space $E$ is $T(M) \times \mathbb{R}$ with co-ordinates $\left(x^{1}, \ldots, x^{n}, \dot{x}^{1}, \ldots, \dot{x}^{n}, t\right)$.

The classical trajectories of the system are the projections of the integral curves (curves on $T(M) \times \mathbb{R}$ ) onto $M$ of the vector field

$$
\Gamma=\dot{x}^{i} \frac{\partial}{\partial x^{i}}+\Lambda^{i} \frac{\partial}{\partial \dot{x}^{i}}+\frac{\partial}{\partial t}
$$

where the $\Lambda^{i}$ satisfy the Euler-Lagrange equations

$$
\frac{\partial^{2} L}{\partial \dot{x}^{i} \partial \dot{x}^{j}} \Lambda^{j}=\frac{\partial L}{\partial x^{i}}-\frac{\partial^{2} L}{\partial \dot{x}^{i} \partial x^{j}} x^{j}-\frac{\partial^{2} L}{\partial \dot{x}^{i} \partial t}
$$

(the Lagrangian is taken to be regular).

Alternatively, $\Gamma$ is the (normalized) characteristic vector field of the exterior derivative of the Cartan form 


$$
\theta(L)=L d t+\frac{\partial L}{\partial \dot{x}^{i}}\left(d x^{i}-\dot{x}^{i} d t\right)
$$

that is,

$$
\Gamma\lrcorner d \theta(L)=0
$$

$$
\langle\Gamma, d t\rangle=1 \text { (normalization), }
$$

where $\downarrow$ denotes the contraction of vectors and forms and $($,$\rangle is the$ natural pairing function. This completely identifies $\Gamma$ with (1.1) since (1.4) requires that

$$
\begin{aligned}
& \left\langle\Gamma, d x^{i}-\dot{x}^{i} d t\right\rangle=0, \\
& \left\langle\Gamma, d \dot{x}^{i}-\Lambda^{i} d t\right\rangle=0,
\end{aligned}
$$

where again the $\Lambda^{i}$ satisfy the Euler-Lagrange equations (1.2).

The relations (1.5), (1.6), suggest a natural local basis of one-forms on $E$, namely $\left\{d x^{i}-\dot{x}^{i} d t, d \dot{x}^{i}-\Lambda^{i} d t, d t\right\}$, dual to the natural basis of vectors $\left\{\partial / \partial x^{i}, \partial / \partial \dot{x}^{i}, \Gamma\right\}$. As a useful illustration of calculations involving these bases consider a vectorfield $X$ on $T(M) \times \mathbb{R}$ with components $\left(\xi^{i}, \eta^{i}, \tau\right)$ relative to the local co-ordinate basis $\left\{\partial / \partial x^{i}, \partial / \partial \dot{x}^{i}, \partial / \partial t\right\}$. Then, relative to the natural basis, the components are given by

$$
X=\left\langle x, d x^{i}-\dot{x}^{i} d t\right\rangle \frac{\partial}{\partial x^{i}}+\left\langle x, d \dot{x}^{i}-\Lambda^{i} d t\right\rangle \frac{\partial}{\partial \dot{x}^{i}}+\langle X, d t\rangle \Gamma
$$

or

$$
X=\left(\xi^{i}-\dot{x}^{i} \tau\right) \frac{\partial}{\partial x^{i}}+\left(\eta^{i}-\Lambda^{i} \tau\right) \frac{\partial}{\partial \dot{x}^{i}}+\tau \Gamma
$$

Similarly a one-form on $E$,

$$
\alpha=\rho_{i} d x^{i}+\sigma_{i} \cdot \dot{x}^{i}+\omega d t,
$$

appears as

$$
\alpha=\left\langle\frac{\partial}{\partial x^{i}}, \alpha\right\rangle\left(d x^{i}-\dot{x}^{i} d t\right)+\left\langle\frac{\partial}{\partial \dot{x}^{i}}, \alpha\right\rangle\left(d \dot{x}^{i}-\Lambda^{i} d t\right)+\langle\Gamma, \alpha\rangle d t,
$$


or

$$
\alpha=\rho_{i}\left(d x^{i}-\dot{x}^{i} d t\right)+\sigma_{i}\left(d \dot{x}^{i}-\Lambda^{i} d t\right)+\left(\dot{x}^{i} \rho_{i}+\Lambda^{i} \sigma_{i}+\omega\right) d t .
$$

In particular if $\alpha=d f$ is exact then

$$
d f=\frac{\partial f}{\partial x^{i}}\left(d x^{i}-\dot{x}^{i} d t\right)+\frac{\partial f}{\partial \dot{x}^{i}}\left(d \dot{x}^{i}-\Lambda^{i} d t\right)+\Gamma(f) d t
$$

Returning to the Cartan form we note that if $L$ and $L^{\prime}$ are equivalent Lagrangians for a given dynamical system inasmuch as

$$
d \theta(L)=d \theta\left(L^{\prime}\right),
$$

then locally there is a function $f: E \rightarrow \mathbb{R}$ such that

$$
\theta\left(L^{\prime}\right)=\theta(L)+d f \text {. }
$$

Since both $\theta(L)$ and $\theta\left(L^{\prime}\right)$ are independent of $\left(\dot{d x}^{i}-\Lambda^{i} d t\right)$ then so is df and (1.9) requires that the values of $f$ be independent of $\dot{x}^{i}$. Thus (1.11) corresponds to the usual gauge variance of the Lagrangian

$$
L^{\prime}=L+\dot{f} .
$$

At this stage it is worth pointing out some of the limitations of this Cartan form approach. Firstly it is not apparent under what circumstances, if any, equations (1.4) and (1.5) provide a two-form $d \theta(L)$ given a vector field $\Gamma$ for the dynamical system. This is essentially the inverse problem in Lagrangian mechancis and I will not address it here. Secondly, and related to this first point, is the possibility that $\Gamma$ may be characteristic of the exterior derivative of a Cartan form $\theta\left(L^{*}\right)$ where $d \theta(L) \neq d \theta\left(L^{*}\right)$. This raises the question of Lagrangians equivalent in a sense different to that of equation (1.12). I will take up this question in a later section.

Finally, the following formulae involving lie derivatives will be useful.

The Lie derivative of a $p$-form $\Omega$ with respect to a vector field $X$ is given by

$$
\left.\left.L_{X} \Omega=d(X\lrcorner \Omega\right)+X\right\lrcorner d \Omega
$$

in particular for a one-form $\alpha$, 


$$
\left.L_{X} \alpha=d\langle X, \alpha\rangle+X\right\lrcorner d \alpha
$$

Furthermore,

$$
L_{X}\left(\Omega_{1} \wedge \Omega_{2}\right)=\left(L_{X} \Omega_{1}\right) \wedge \Omega_{2}+\Omega_{1} \wedge L_{X} \Omega_{2}
$$

and

$$
L_{X}(d \Omega)=d\left(L_{X} \Omega\right)
$$

for any $p$-forms $\Omega, \Omega_{1}$ and $q$-form $\Omega_{2}$. Last, but not least, for vector fields $X, Y$,

$$
L_{X} Y=[X, Y]
$$

\section{Invariance groups and conservation laws}

The general theory of invariance groups and conservation laws is based on the following two ideas.

(i) Any one parameter group on $T(M) \times \mathbb{R}$ which permutes the integral curves of $\Gamma$ is called an invariance group of the system. The local condition that a group generated by a vector field $X$ on $T(M) \times \mathbb{R}$ be an invariance group is

$$
\begin{gathered}
{[X, \Gamma]=\lambda \Gamma} \\
(\lambda: T(M) \times \mathbb{R} \rightarrow \mathbb{R}) .
\end{gathered}
$$

(More details are given later and in the Appendix, suffice it to note here that the idea of looking at invariance groups on $T(M) \times \mathbb{R}$ (the so-called "velocity-dependent" transformations) is due to Cartan and grew out of Lie's theory of ordinary differential equations where the invariance groups are on $M \times \mathbb{R}$. )

(ii) A function $F: T(M) \times \mathbb{R} \rightarrow \mathbb{R}$ is conserved along the classical trajectories if

$$
\Gamma(F)=0 \text {. }
$$

Comparing the expression (1.9) for $d F$ with (2.2) it is clear that $F$ is a constant of the motion if $d F$ does not involve $d t$ when described relative to the natural basis for $T^{*}(E)$; clearly with this choice of basis the classical idea of an ignorable co-ordinate is quite unambiguous. 
The thrust of the neoclassical Lie theory of differential equations, Noether's Theorem and of the Cartanian approach, is to relate conservation laws and invariance groups. To this end it is useful to classify the various cases of (2.1). The following results are more or less classical (see in part, Crampin [1], Sarlet and Cantrijn [7] and Prince [5]).

A. A Lie symmetry of the system is a one-parameter group on $M \times \mathbb{R}$ generated locally by a vector field

$$
\chi=\xi^{i} \frac{\partial}{\partial x^{i}}+\tau \frac{\partial}{\partial t}
$$

$\left(\xi^{i}, \tau: M \times \mathbf{R} \rightarrow \mathbb{R}\right)$. In this case (2.1) is just

$$
\left[X^{(1)}, \Gamma\right]=-\dot{\tau} \Gamma
$$

where

$$
x^{(I)}=x+\left(\dot{\xi}^{i}-\dot{x}^{i} \dot{\tau}\right) \frac{\partial}{\partial \dot{x}^{i}}
$$

is the first prolongation of $X$. (from $M \times \mathbb{R}$ to $T(M) \times \mathbb{R}$; see the Appendix).

These one-parameter groups are finite in number, say $r$ of them, and form an $r$-parameter lie groups, denoted $G$, on $M \times \mathbb{R}$. In particular the associated vector fields $X_{1}, \ldots, X_{r}$ form an $r$-dimensional Lie algebra, denoted $A$.

In general conservation laws are obtainable from the Lie algebra and the transformation properties $X^{(1)}(F)=0, \Gamma(F)=0$ (see Prince [5]), although not in closed form and there is not a one-to-one association of conservation laws with Lie symmetries, for example, the cases $X_{i}^{(1)}(F)=X_{j}^{(1)}(F)=0$ and $X^{(1)}\left(F_{i}\right)=X^{(1)}\left(F_{j}\right)=0$, may occur.

The theory of Lie symmetries is, in fact, the modern version of Lie's theory of ordinary differential equations and transcends questions of variational principles.

B. A Noether symmetry of the system is a one-parameter group belonging to $G$ with generator $Y \in A$ and the additional property that

$$
L_{Y}(1) \theta(L)=d f
$$


$(f: M \times \mathbb{R} \rightarrow \mathbb{R})$. This gives a closed form conservation law

$$
F=f-\left\langle Y^{(1)}, \theta(L)\right\rangle
$$

with the transformation property

$$
Y^{(I)}(F)=0 \text {. }
$$

In general, however, not all conservation laws are obtainable in this way (see Case D below).

I should point out that this presentation of Noether symmetries is novel in two ways. Firstly, Noether symmetries usually arise as oneparameter groups on $M \times \mathbb{R}$ which leave the Lagrangian invariant as opposed to groups leaving the variational principle invariant; thus it is not immediately obvious that (2.1) is satisfied. Secondly, Noether symmetries are usually formulated locally so that

$$
L_{Y}(1)(L d t)-d f \in C
$$

where $C$ is the subspace of $T^{*}(E)$ spanned by the contact forms $d x^{i}-\dot{x}^{i} d t$ (see Crampin [1]). The formulation (2.6) is equivalent and is needed to demonstrate the Noether symmetries are a subcase of Cartan symmetries (see Case $\mathrm{D}$ below).

C. A dynamical symmetry of the system is just the most general case of (2.1). It is a one-parameter group on $T(M) \times \mathbb{R}$ (as opposed to $M \times \mathbb{R}$ ) generated locally by a vector field

$$
X=\xi^{i} \frac{\partial}{\partial x^{i}}+n^{i} \frac{\partial}{\partial \dot{x}^{i}}+\tau \frac{\partial}{\partial t}
$$

$\left(\xi^{i}, n^{i}, \tau: T(M) \times \mathbb{R} \rightarrow \mathbb{R}\right)$ with the properties that

$$
\begin{aligned}
{[x, \Gamma] } & =-\Gamma(\tau) \Gamma, \\
n^{i} & =\Gamma\left(\xi^{i}\right)-\dot{x}^{i} \Gamma(\tau), \\
x\left(\Lambda^{i}\right) & =\Gamma\left(\eta^{i}\right)-\Lambda^{i} \Gamma(\tau) .
\end{aligned}
$$

It is important to realise that the action of a dynamical symmetry is to permute the classical trajectories on $M \times \mathbb{R}$ but not in general as a one-parameter group on $M \times \mathbf{R}$. (This only occurs when a vector field 
generating the group can be found which projects onto $M \times \mathbb{R}$ ). For this reason the collection of dynamical symmetries do not form a Lie algebra and, unlike Lie symmetries, it is not usually possible to find the form of all the vector fields $X$.

D. A Cartan symmetry is a dynamical symmetry generated by a vector field $Y$ with the additional property

$$
L_{Y} \Theta(L)=d f
$$

$(f: T(M) \times \mathbf{R} \rightarrow \mathbf{R})$. This gives a closed form conservation law

$$
F=f-\langle Y, \Theta(L)\rangle
$$

and the transformation property

$$
Y(F)=0 .
$$

These results derive from the following theorem.

THEOREM 1. For each point $P$ in evolution space $\left\langle\Gamma_{p}, \alpha\right\rangle=0$ if and only if there exists a vector $X \in T_{p}(E)$ such that $\left.\alpha=X\right\lrcorner d \Theta_{p}(L)$.

Proof. Consider the linear map

$$
\left.S: T_{p}(E) \rightarrow T_{p}(E), S(X)=X\right\lrcorner d \Theta_{p}(L) .
$$

The null and rank spaces of $S$ are

$$
\begin{aligned}
& N_{S}=\left\{X \in T_{p}(E): X=f T, f: T_{p}(M) \times \mathbb{R} \rightarrow \mathbb{R}\right\}, \\
& \left.R_{S}=\left\{\alpha \in T_{p}^{*}(E): \alpha=X\right\lrcorner d \Theta_{p}(L), X \in T_{p}(E)\right\},
\end{aligned}
$$

respectively, thus $\operatorname{dim} R_{S}=\operatorname{dim} T_{p}(E)-\operatorname{dim} N_{S}=2 n$. Now if $\alpha \in R_{S}$ then

$$
\left.\left\langle\Gamma_{p}, \alpha\right\rangle=\left(\Gamma_{p}, X\right\lrcorner d \Theta_{p}(L)\right)=d \Theta\left(X, \Gamma_{p}\right)=0
$$

so that

$$
R_{S} \subseteq\left\{\alpha \in T_{p}^{*}(E):\left\langle\Gamma_{p}, \alpha\right\rangle=0\right\}
$$

As this subspace has dimension $2 n$ and since both spaces are generated by $\left\{d x^{i}-\dot{x}^{i} d t, \dot{d x^{i}}-\Lambda^{i} d t\right\}$,

$$
R_{S}=\left\{\alpha \in T_{p}^{*}(E):\left\langle\Gamma_{p}, \alpha\right\rangle=0\right\} .
$$


The other important results follow as corollaries.

COROLLARY. (i) If $L_{Y} \Theta(L)=d f$ for some vector field $Y$ then $f-\langle y, \Theta(L)\rangle$ is a constant of the motion.

(ii) If $F$ is a constant of the motion then there is a vector field $Y$ such that $L_{Y} \Theta(L)=d(F+\langle Y, \Theta(L)\rangle)$ and any vector field $Y+g \Gamma(g: T(M) \times R \rightarrow R)$ has the same property.

Proof. From Theorem 1 a constant of the motion is a one-form $Y\lrcorner d \Theta(L)$ which is exact. Hence

$$
\left.L_{Y} \Theta(L)=Y\right\lrcorner d \Theta(L)+d(Y, \Theta(L))
$$

is exact. Results $(i)$ and $(i i)$ follow immediately.

Clearly Noether symmetries (B) are a special case of Cartan symmetries. The latter differ from the former in that there is a one-toone correspondence between all the conservation laws and the set of "normalized" Cartan symmetries (normalized so that $[Y, \Gamma]=0$ ). However, while it is possible to find all the Noether symmetries, the fact that the Cartan symmetries do not form a Lie algebra means that it may not be possible to find all of them (and hence all the conservation laws).

\section{Invariance groups - towards a classification}

The results A-D of the last section suggest a possible classification scheme for dynamical symmetries using the transformation properties of $\theta(L)$ under the action of the groups. For Cartan (and Noether) symmetries $\Theta(L)$ transforms according to

$$
L_{X} \Theta(L)=d f \Rightarrow L_{X} d \Theta(L)=0 .
$$

The corresponding result for Lie symmetries is given in the following theorem (see Prince [5] and also Lutzky [3] and Marmo and Saletan [4]).

THEOREM 2. If a vector field $X$ on $M \times \mathbf{R}$ generates a Lie symmetry then

$$
L_{X}(1)^{\Theta(L)}=\Theta\left(L^{*}\right)+d f
$$

where $f: M \times \mathbb{R} \rightarrow \mathbb{R}$,. 


$$
L^{*}+\dot{f}=X^{(I)}(L)+L \dot{\tau}
$$

and

$$
\Gamma\lrcorner d \theta\left(L^{*}\right)=0 .
$$

Proof. Taking $X^{(1)}$ to be given by (2.5) gives

$$
\text { (3.5) } \begin{aligned}
L_{X}(I) & \Theta(L)=\left(X^{(I)}(L)+L \dot{\tau}\right) d t \\
& +\left[L \frac{\partial \tau}{\partial x^{i}}+X^{(I)}\left(\frac{L}{\partial \dot{x}^{i}}\right)+\frac{\partial L}{\partial \dot{x}^{j}}\left(\frac{\partial \xi^{j}}{\partial x^{i}}-\dot{x}^{j} \frac{\partial \tau}{\partial x^{i}}\right)\right]\left(d x^{i}-\dot{x}^{i} d t\right) .
\end{aligned}
$$

Now

$$
L \frac{\partial \tau}{\partial x^{i}}+X^{(\lambda)}\left(\frac{\partial L}{\partial x^{i}}\right)+\frac{\partial L}{\partial \dot{x}^{j}}\left(\frac{\partial \xi^{j}}{\partial x^{i}}-\dot{x}^{j} \frac{\partial \tau}{\partial x^{i}}\right)=\frac{\partial}{\partial \dot{x}^{i}}\left(X^{(I)}(L)+L \dot{\tau}+g\right),
$$

where $g: M \times \mathbb{R} \rightarrow \mathbb{R}$. Hence, including the gauge variance arising from $g,(3.5)$ can be written

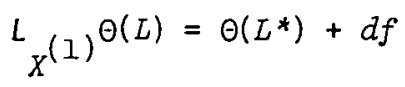

where

$$
L^{*}+\dot{f}=X^{(l)}(L)+L \dot{\tau}
$$

and $f: M \times R \rightarrow \mathbb{R}$.

Moreover,

$$
\begin{aligned}
& \left.\left.\left.0=L_{X}(1)(\Gamma\lrcorner d \Theta(L)\right)=\left[X^{(1)}, \Gamma\right]\right\lrcorner d \theta(L)+\Gamma\right\lrcorner L_{X}(1) d \Theta(L) \\
& \Rightarrow 0=\Gamma\lrcorner d \Theta\left(L^{*}\right)
\end{aligned}
$$

by virtue of (2.4) and (3.7).

There are a number of remarks.

(i) A converse to this result does not hold, namely, given a pair of equivalent Lagrangians $L, L^{*}$, we cannot, in general, obtain an $X \in A$ using (3.3) (equivalent Lagrangians for the free particle and harmoric oscillator provide counterexamples).

(ii) It is not clear whether a closed form conserved quantity can be constructed from (3.2) although in the case of the so-called "higher-order 
Noether-symmetries" of Sarlet and Cantrijn [8], this can be done (Prince [6]).

Turning to the more general case of dynamical symetries we have

THEOREM 3. If $X$ generates a dynamical symmetry then

$$
L_{X} \Theta(L)=\Theta\left(L^{*}\right)+d f
$$

with

$$
\Gamma\lrcorner d \Theta\left(L^{*}\right)=0
$$

$(f: T(M) \times \mathbb{R} \rightarrow \mathbb{R})$ if and only if

$$
S_{[i j]}=0
$$

where

$$
S_{i j}=\frac{\partial^{2} L}{\partial \dot{x}^{i} \partial \dot{x}^{k}}\left(\frac{\partial \xi^{k}}{\partial \dot{x}^{j}}-\dot{x}^{k} \frac{\partial \tau}{\partial \dot{x}^{j}}\right) .
$$

Proof. Taking $X$ to be of the form (2.10) gives

(3.14) $L_{X} \Theta(L)=(X(L)+L \Gamma(\tau)) d t$

$$
\begin{aligned}
+\left[L \frac{\partial \tau}{\partial x^{i}}+X\left(\frac{\partial L}{\partial \dot{x}^{i}}\right)+\frac{\partial L}{\partial \dot{x}^{j}}\left(\frac{\partial \xi^{j}}{\partial x^{i}}-\dot{x}^{j} \frac{\partial \tau}{\partial x^{i}}\right)\right]\left(d x^{i}-\dot{x}^{i} d t\right) \\
+\left[L \frac{\partial \tau}{\partial \dot{x}^{i}}+\frac{\partial L}{\partial \dot{x}^{j}}\left(\frac{\partial \xi^{j}}{\partial \dot{x}^{i}}-\dot{x}^{j} \frac{\partial \tau}{\partial \dot{x}^{i}}\right)\right]\left(\dot{x}^{i}-\Lambda^{i} d t\right)
\end{aligned}
$$

Attempting a solution of the form (3.10) requires that

$$
\begin{aligned}
X(L)+L \Gamma(\tau) & =L^{*}+\Gamma(f), \\
L \frac{\partial \tau}{\partial x^{i}}+X\left(\frac{\partial L}{\partial \dot{x}^{i}}\right)+\frac{\partial L}{\partial x^{j}}\left(\frac{\partial \xi^{j}}{\partial x^{i}}-\dot{x^{j}} \frac{\partial \tau}{\partial x^{i}}\right) & =\frac{\partial L^{*}}{\partial \dot{x}^{i}}+\frac{\partial f}{\partial x^{i}}, \\
L \frac{\partial \tau}{\partial \dot{x}^{i}}+\left(\frac{\partial L}{\partial \dot{x}^{j}}\right)\left(\frac{\partial \xi^{j}}{\partial \dot{x}^{i}}-\dot{x^{j}} \frac{\partial \tau}{\partial \dot{x}^{i}}\right) & =\frac{\partial f}{\partial \dot{x}^{i}} .
\end{aligned}
$$

A necessary and sufficient condition that such a solution exists is surprisingly just the integrability of $f$, namely

$$
\frac{\partial^{2} f}{\partial \dot{x}^{i} \partial \dot{x}^{j}}=\frac{\partial^{2} f}{\partial \dot{x}^{j} \partial \dot{x}^{i}}
$$


which, in the light of the last of equations (3.15), is (3.12) where $S_{i j}$ is given by (3.13). If $(3.10)$ is true then (3.11) follows analogously to (3.4).

The condition (3.12) is not in general satisfied by all dynamical symmetries (the two-dimensional free particle is a good counterexample). Indeed it is not even possible to find a function $g: T(M) \times \mathbb{R} \rightarrow \mathbb{R}$ such that $X+g \Gamma$ satisfies (3.12) for any dynamical symmetry $X$ (the transformation $X \rightarrow X+g \Gamma$ preserves transformation properties like $X(F)=0$ for a conservation law $F$ ). However, the transformation $X \rightarrow X-(X, d t) \Gamma$ does simplify (3.12) and (3.13):

$$
S_{[i j]}^{\prime}=0
$$

where

$$
S_{i j}^{\prime}=\frac{\partial^{2} L}{\partial \dot{x}^{i} \partial \dot{x}^{k}} \frac{\partial \xi^{, k}}{\partial \dot{x}^{j}}, \quad \xi^{, k}=\xi^{k}-\dot{x}^{k} \tau .
$$

Condition (3.12) is met by the dynamical symmetries of all one-dimensional systems and when $X$ is a Lie or Cartan symmetry.

It is an open question whether or not any pair of equivalent Lagrangians lead to a dynamical symmetry through the first of equations (3.15). It is also unclear whether dynamical symmetries satisfying Theorem 3 lead to closed form constants of motion.

In conclusion, I have developed an almost complete classification of dynamical symmetries through the transformation property (3.10). Most importantly, the manifestly geometric Lie symmetries bave the transformation property (3.2).

\section{Example - the Kepler problem}

For the sake of illustration I will consider the Kepler problem in two dimensions with local co-ordinates $r=\left(x^{1}, x^{2}\right)$ for $M$. Taking

$$
\begin{aligned}
& L=\frac{1}{2}\left(\dot{x}^{1^{2}+\dot{x}^{2}}\right)+\mu / r \quad(\mu>0), \\
& \Gamma=x^{i} \frac{\partial}{\partial x^{i}}-\frac{\mu x^{i}}{r^{3}} \frac{\partial}{\partial \dot{x}^{i}}+\frac{\partial}{\partial t}
\end{aligned}
$$


and local bases for $T(E), T^{*}(E)$ as

$$
\left\{\frac{\partial}{\partial x^{i}}, \frac{\partial}{\partial \dot{x}^{i}}, \Gamma\right\},\left\{d x^{i}-\dot{x}^{i} d t, d \dot{x}^{i}-\Lambda^{i} d t, d t\right\}
$$

where $\Lambda^{i}=-\mu x^{i} / x^{3}$, the exterior derivative of the Cartan form is

$$
\text { (4.3) } \quad d \theta(L)=\delta_{i j}\left(d \dot{x}^{i}-\Lambda^{i} d t\right) \wedge\left(d x^{j}-\dot{x}^{j} d t\right) .
$$

The first prolongations of the three Lie symmetries are

$$
\begin{aligned}
& x_{1}^{(1)}=-\dot{x}^{i} \frac{\partial}{\partial x^{i}}-\Lambda^{i} \frac{\partial}{\partial \dot{x}^{i}}+\Gamma \\
& x_{2}^{(1)}=-x^{2} \frac{\partial}{\partial x^{1}}+x^{1} \frac{\partial}{\partial x^{2}}-\dot{x}^{2} \frac{\partial}{\partial \dot{x}^{1}}+\dot{x}^{1} \frac{\partial}{\partial \dot{x}^{2}}
\end{aligned}
$$

and

$$
x_{3}^{(1)}=\left(\frac{2}{3} x^{i}-\dot{x}^{i} t\right) \frac{\partial}{\partial x^{i}}-\left(\frac{1}{3} \dot{x}^{i}+\Lambda^{i} t\right) \frac{\partial}{\partial \dot{x}^{i}}+t \Gamma .
$$

Using the integration procedures in Prince [5], the Lie algebra $\left\{x_{1}, x_{2}, x_{3}\right\}$ leads to the conservation laws:

Energy

$$
\begin{aligned}
& E=\left(\dot{x}^{2}+\dot{x}^{2}\right)-\frac{\mu}{r}, \\
& h=x_{1} \dot{x}_{2}-x_{2} \dot{x}_{1},
\end{aligned}
$$

(4.5) Angular Momentum

Runge-Lenz vector $\left(x^{i}\right.$ component $) R^{i}=x^{i}(\dot{r} \cdot \dot{r})-\dot{x}^{i}(r \cdot \dot{r})-\frac{\mu x^{i}}{r}$. Now $X_{1}, X_{2}$ generate Noether symmetries and $E, h$ are recoverable from

$$
L_{X}(1) \Theta(L)=d f \text { and } F=f-\left(X^{(1)}, \Theta(L)\right)
$$

however

$$
L_{X_{3}}(1) \Theta(L)=\frac{1}{3} \Theta(L)
$$

so that $L^{*}=\frac{1}{3} L$ and there are no Noether symmetries corresponding to $R^{l}$ and $R^{2}$. Using the last of (4.5) and (2.13) we can construct (up to a 
multiple of $\Gamma$ ) the Cartan symmetries corresponding to $R^{1}, R^{2}$ respectively:

$$
\begin{aligned}
y_{1}=\left(x^{2} \dot{x}^{1}+x^{2} \dot{x}^{2}\right) \frac{\partial}{\partial x^{1}}+\left(x^{2} \dot{x}^{1}-x^{1} \dot{x}^{2}\right) & \frac{\partial}{\partial x^{2}} \\
& +\left(\dot{x}^{2}-\frac{\mu}{r}\right) \frac{\partial}{\partial \dot{x}^{1}}-\dot{x}^{1} \dot{x}^{2} \frac{\partial}{\partial \dot{x}^{2}}+x^{1} \frac{\partial}{\partial t},
\end{aligned}
$$

$(4.7)$

$$
\begin{aligned}
y_{2}=\left(x^{1} \dot{x}^{2}-x^{2} \dot{x}^{1}\right) \frac{\partial}{\partial x^{1}}+\left(x^{1} \dot{x}^{1}+x^{2} \dot{x}^{2}\right) & \frac{\partial}{\partial x^{2}} \\
& -\dot{x}^{1} \dot{x}^{2} \frac{\partial}{\partial \dot{x}^{1}}+\left(\dot{x}^{1}-\frac{\mu}{x}\right) \frac{\partial}{\partial \dot{x}^{2}}+x^{2} \frac{\partial}{\partial t} .
\end{aligned}
$$

The set of four Cartan symmetries does not form a Lie algebra on $T(M) \times \mathbb{R}$, the commutators being

$$
\begin{aligned}
{\left[X_{1}^{(1)}, Y_{1}\right] } & =\left[X_{1}^{(1)}, Y_{2}\right]=0, \\
{\left[X_{2}^{(1)}, Y_{1}\right] } & =-Y_{2},\left[X_{2}^{(1)}, Y_{2}\right]=Y_{1}, \\
{\left[Y_{1}, Y_{2}\right] } & =2\left(h X_{1}^{(1)}+E X_{2}^{(1)}\right) .
\end{aligned}
$$

However, the set is useful inasmuch as the transformation properties

$$
\begin{aligned}
& X_{1}^{(I)}(E)=X_{1}^{(1)}(h)=X_{I}^{(I)}\left(R^{i}\right)=0, \\
& X_{2}^{(I)}(E)=0, \quad X_{2}^{(I)}(h)=0, X_{2}^{(l)}\left(R^{l}\right)=-R^{2}, X_{2}^{(I)}\left(R^{2}\right)=R^{\perp},
\end{aligned}
$$

$$
\begin{aligned}
& y_{1}(E)=Y_{2}(E)=0, \\
& y_{1}(h)=-R^{2}, \quad Y_{2}(h)=R^{7}, \\
& Y_{1}\left(R^{2}\right)=-2 E h, \quad Y_{2}\left(R^{1}\right)=2 E h,
\end{aligned}
$$

indicate that the "basis of conservation laws" associated with the set may be taken as $\{E, h\}$. In contrast, when the Lie algebra is used, the basis is $\left\{E, h, R^{1}, R^{2}\right\}$. Finally, the transformation properties of the Cartan symmetries $Y_{1}$ and $Y_{2}$ under the ("degenerate") Lie symmetry $X_{3}$ are interesting although not surprising: 


$$
\left[X_{3}, Y_{a}\right]=\frac{1}{3} y_{a}
$$

\section{Appendix}

One of the basic technical questions in Lie's group theoretic approach to differential equations concerns the extension of a group action on $M \times \mathbb{R}$ to one on $T(M) \times \mathbb{R}$. Rather interestingly a basic technical question arising in the Cartanian approach to Lagrangian systems used here is "Under what circumstances does a one-parameter group on $T(M) \times \mathbb{R}$ 'project' onto a one-parameter group on $M \times \mathbb{R}$ ?"

Before starting with the details a few points need to be kept in mind. Firstly, while the natural place for trajectories of a dynamical system is $M$, the action of a one-parameter group on the solutions takes place on $M \times \mathbb{R}$, part of the action being a re-parametrisation of the Newtonian time along the trajectories. Consequently it will be necessary to extend the solutions on $M$ to curves on $M \times \mathbb{R}$. Secondly, because $T(M) \times \mathbb{R} \neq T(M \times \mathbb{R})$ we will not need the usual lift from $M$ to $T(M)$ but two lifts, one from $M$ to $T(M) \times \mathbb{R}$ and one from $M \times \mathbb{R}$ to $T(M) \times \mathbb{R}$. The technical aspects make the notation a little unwieldly but the intuitive ideas can still be clearly expressed.

Consider a member of a family of curves on $M$,

$$
\gamma: \mathbb{R} \rightarrow M, \quad \gamma(\lambda)=P, \mu(P)=\left(x^{i}\right)
$$

( $\mu$ is a chart for an open region of $M$ containing $P$ ). I will denote the corresponding curve on $M \times \mathbb{R}$ by $\tilde{\gamma}$ :

$$
\tilde{\gamma}: \mathbb{R} \rightarrow M \times \mathbb{R}, \tilde{\gamma}(\lambda)=(P, \lambda), \tilde{\mu}(P, \lambda)=\left(x^{i}, \lambda\right),
$$

where $\tilde{\mu}$ corresponds to $\mu$ in an obvious way. Now, if the family of curves on $M$ has tangent vector field $X$, then $\gamma$ and $\tilde{\gamma}$ are lifted to the same curve on $T(M) \times \mathbb{R}$ by the mappings $T, \tilde{T}$ respectively:

$$
\begin{aligned}
& T: C^{\infty}(\mathbb{R}, M) \rightarrow C^{\infty}(\mathbb{R}, T(M) \times \mathbb{R}), \\
& \tilde{T}: C^{\infty}(\mathbb{R}, M \times \mathbb{R}) \rightarrow C^{\infty}(\mathbb{R}, T(M) \times \mathbb{R}), \\
& \gamma+\hat{\gamma}: T(\gamma)(\lambda)=\hat{\gamma}(\lambda)=\left(P=\gamma(\lambda), X_{p}, \lambda\right), \\
& \tilde{\gamma} \rightarrow \hat{\gamma}: \tilde{T}(\tilde{\gamma})(\lambda)=\hat{\gamma}(\lambda) ;
\end{aligned}
$$


and

$$
\hat{\mu}\left(P, x_{p}, \lambda\right)=\left(x^{i}, \frac{d x^{i}}{d \lambda}, \lambda\right)
$$

is the obvious chart for $T(M) \times \mathbb{R}$.

$$
\begin{gathered}
\text { A one-parameter }(a \in I \subseteq \mathbb{R}) \text { group } \psi \text { on } M \times \mathbb{R} \text { is denoted } \\
\psi_{a}: M \times \mathbb{R} \rightarrow M \times \mathbb{R}
\end{gathered}
$$

where

(A4)

$$
\begin{gathered}
\psi:(M \times \mathbb{R}) \times I+M \times \mathbb{R} ; \\
\psi(P, \nu ; a)=\psi_{a}(P, \nu)=(\bar{P}, \bar{v}) .
\end{gathered}
$$

It is important to realise that both $\bar{P}$ and $\bar{v}$ depend on each of $P, v$ and $a$.

The image of $\tilde{\gamma}$ under $\psi_{a}$ can now be written $\bar{\gamma}=\psi_{a} \circ \tilde{\gamma}$. The oneparameter group can be used to define a function

$$
\tilde{\psi}_{a}: C^{\infty}(\mathbb{R}, M \times \mathbb{R}) \rightarrow C^{\infty}(\mathbb{R}, M \times \mathbb{R})
$$

by

$$
\bar{\gamma}(\bar{\lambda})=\tilde{\psi}_{a}(\tilde{\gamma})(\bar{\lambda})=\left(\psi_{a} \circ \tilde{\gamma}\right)(\lambda)
$$

where the new curve parameter $\bar{\lambda}$ depends on $\lambda$ through (A6)

$$
\tilde{\gamma}(\lambda)=(P, \lambda) \text { and }\left(\psi_{a} \circ \tilde{\gamma}\right)(\lambda)=(\bar{P}, \bar{\lambda}) \text {. }
$$

The first extension of $\psi_{a}, \psi_{a}^{(1)}: T(M) \times \mathbb{R} \rightarrow T(M) \times \mathbb{R}$ can now be implicitly defined as follows :

$$
\tilde{\psi}_{a}^{(1)}(T(\gamma))=\tilde{T}\left(\tilde{\psi}_{a}(\tilde{\gamma})\right) \quad(\text { for all } \gamma \text { on } M)
$$

where

$$
\tilde{\Psi}_{a}^{(1)}: C^{\infty}(\mathbb{R}, T(M) \times \mathbb{R}) \rightarrow C^{\infty}(\mathbb{R}, T(M) \times \mathbb{R})
$$

is defined similarly to $\tilde{\psi}_{a}$ above, namely,

$$
\tilde{\psi}_{a}^{(1)}(\hat{\gamma})(\bar{\lambda})=\left(\psi_{a}^{(1)} \circ \hat{\gamma}\right)(\lambda)
$$


Thus $\psi_{a}^{(1)}$ is the mapping that completes the following diagram for any curve $\gamma$ on $M$,

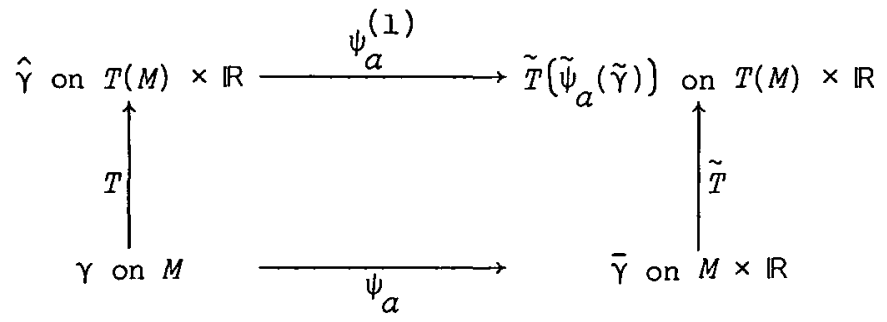

The definition (A7) of $\psi_{a}^{(1)}$ does not specify its action on all points of $T(M) \times \mathbb{R}$ however, this can be done in a natural way (for example by considering a congruence of curves $\gamma$ on $M$ ) and it can then be shown that $\psi_{a}^{(l)}$ is a one-parameter group on $T(M) \times \mathbb{R}$. Further it can be shown that, if $\psi_{a}$ is locally generated by the vector field

$$
x=\xi^{i} \frac{\partial}{\partial x^{i}}+\tau \frac{\partial}{\partial t}
$$

then $\psi_{a}^{(1)}$ is generated by

$$
X^{(1)}=\xi^{i} \frac{\partial}{\partial x^{i}}+\left(\dot{\xi}^{i}-\dot{x}^{i} \dot{\tau}\right) \frac{\partial}{\partial \dot{x}^{i}}+\tau \frac{\partial}{\partial t} .
$$

$X^{(1)}$ is called the first extension or prolongation of $X$.

Conversely, if $\phi_{a}$ is a one-parameter group on $T(M) \times \mathbb{R}$ then it is the first extension of a one-parameter group on $M \times \mathbb{R}$ if and only if

$$
\left(\exists \psi_{a} \text { on } M \times \mathbb{R}\right)(\forall \gamma \text { on } M) \tilde{\phi}_{a}(T(\gamma))=T\left(\tilde{\psi}_{a}(\tilde{\gamma})\right) \text {. }
$$

$\phi_{a}$ is then said to project onto $M \times \mathbb{R}$. In particular, if $\phi_{a}$ is generated by a vector field $Z$ on $T(M) \times \mathbb{R}$ then the equivalent infinitesimal condition to (All) is

$$
Z=h X^{(1)}
$$

for some $X$ on $M \times \mathbb{R}$ and $h: T(M) \times \mathbb{R} \rightarrow \mathbb{R}$.

A related matter is whether or not a one-parameter group $\phi_{a}$ on 
$T(M) \times \mathbb{R}$ will take an arbitrary lifted curve onto another lifted curve, namely,
(Al3)
$(\forall r$ on
$M)(\exists \rho$ on
M) $\tilde{\phi}_{a}(T(\gamma))=T(\rho)$ ?

The infinitesimal equivalent of this is that the vector field $Z$ generating $\phi_{a}$ preserves the set of contact forms

$$
L_{Z}\left(d x^{i}-\dot{x}^{i} d t\right) \in C
$$

Rather surprisingly, for systems with two or more degrees of freedom this requires that $\phi_{a}$ project onto a one-parameter group on $M \times \mathbb{R}$ (see Sarlet and Cantrijn [7]).

Finally I will consider the invariance condition (2.1). The integral curves of $\Gamma$ (on $T(M) \times \mathbb{R}$ ) are lifted from the classical trajectories of the system, the family of these integral curves will be denoted $F$. A cne-parameter group $\phi_{a}$ on $T(M) \times \mathbb{R}$ is defined to be an invariance group of the dynamical system if it permutes the integral curves, that is if

$$
(\forall \gamma \in F) \quad \phi_{a} \circ \gamma \in F \text {. }
$$

The infinitesimal version of this is

$$
L_{Z} \Gamma=\lambda \Gamma
$$

where $Z$ generates $\phi_{\alpha}$ and $\lambda: T(M) \times \mathbb{R} \rightarrow \mathbb{R}$. It is clear from the foregoing that the corresponding action on the classical trajectories will be that of a one-parameter group if and only if $Z$ satisfies (Al2).

\section{References}

[1] M. Crampin, "Constants of the motion in Lagrangian mechanics", Internat. J. Theoret. Phys. 16 (1977), 741-754.

[2] Frank B. Estabrook, "Differential geometry as tool for applied mathematicians", Geometrical approaches to differential equations, 1-22 (Proc. Fourth Scheveningen Conf. Differential Equations, 1979. Lecture Notes in Mathematics, 810. SpringerVerlag, Berlin, Heidelberg, New York, 1980). 
[3] M. Lutzky, "Symmetry groups and conserved quantities for the harmonic oscillator", J. Phys. A: Math. Gen. (2) 11 (1978), 249-258.

[4] G. Marmo and E.J. Saletan, "Ambiguities in the Lagrangian and Hamiltonian formalism: transformation properties", Nuovo Cimento B 40 (1977), 67-89.

[5] Geoffrey Eamonn Prince, "Lie symmetries of differential equations and dynamical systems" (PhD thesis, La Trobe University, Bundoora, Victoria, 1981). See also: Abstract, Bull. Austral. Math. Soc. 25 (1982), 309-311.

[6] Geoff Prince, "A note on the higher-order Noether symmetries of Sarlet and Cantrijn" (Report DIAS-STP-82-28. Dublin Institute of Advanceã Stuåies, Dublin, Eire, 1982).

[7] Willy Sarlet and Frans Cantrijn, "Generalizations of Noether's theorem in classical mechanics", SIAM Rev. 23 (1981), 467-494.

[8] Willy Sarlet and Frans Cantrijn, "Higher-order Noether symmetries and constants of the motion", J. Phys. A: Math. Gen. (2) 14 (1981), 479-492.

Department of Applied Mathematics,

La Trobe University,

Bundoora,

Victoria 3083,

Australia.

Current Address:

School of Theoretical Physics,

Dublin Institute for Advanced Studies,

10 Burlington Road,

Dublin 4 ,

Eire. 